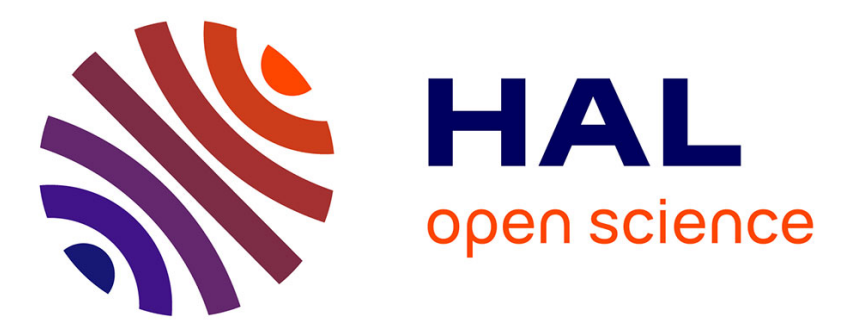

\title{
Antiappétants pour la fourmi attine, Acromyrmex octospinosus (Reich) (Hymenoptera - Formicidae), chez certaines espèces d'igname (Dioscoreaceae) cultivées aux Antilles
}

\author{
Gérard Febvay, Paul Bourgeois, Alain Kermarrec
}

\section{To cite this version:}

Gérard Febvay, Paul Bourgeois, Alain Kermarrec. Antiappétants pour la fourmi attine, Acromyrmex octospinosus (Reich) (Hymenoptera - Formicidae), chez certaines espèces d'igname (Dioscoreaceae) cultivées aux Antilles. Agronomie, 1986, 5 (5), pp.439-444. hal-00884829

\section{HAL Id: hal-00884829 \\ https://hal.science/hal-00884829}

Submitted on 1 Jan 1986

HAL is a multi-disciplinary open access archive for the deposit and dissemination of scientific research documents, whether they are published or not. The documents may come from teaching and research institutions in France or abroad, or from public or private research centers.
L'archive ouverte pluridisciplinaire HAL, est destinée au dépôt et à la diffusion de documents scientifiques de niveau recherche, publiés ou non, émanant des établissements d'enseignement et de recherche français ou étrangers, des laboratoires publics ou privés. 


\title{
Antiappétants pour la fourmi attine, Acromyrmex octospinosus (Reich) (Hymenoptera - Formici- dae), chez certaines espèces d'igname (Diosco- reaceae) cultivées aux Antilles
}

\author{
Gérard FEBVAY, Paul BOURGEOIS (*) \& Alain KERMARREC \\ I.N.R.A., Station de Zoologie et Lutte biologique, Centre de Recherches Antilles-Guyane, Domaine Duclos, \\ F 97170 Petit-Bourg, Guadeloupe \\ (*) Université Antilles Guyane, Laboratoire de Chimie, B.P. 592, F 97167 Pointe-à-Pitre, Guadeloupe
}

RÉSUMÉ

\begin{abstract}
La fourmi attine présente en Guadeloupe, Acromyrmex octospinosus, ne découpe pas les feuilles d'une certaine espèce d'igname (Dioscorea cayenensis) ssp Cayenensis alors que d'autres espèces sont ravagées. Cette préférence est vérifiée au laboratoire. Le refus d'affouragement sur des feuilles de $D$.c. cayenensis est très strict et peut entraîner la mort du nid approvisionné avec ce seul végétal. La résistance est d'ordre chimique et les composés en cause ne sont pas répulsifs mais antiappétants. Ils sont extractibles au méthanol. Les saponines paraissent à l'origine de cette résistance.
\end{abstract}

Mots clés additionnels : Fourmis champignonnistes, Dioscorea, relations plantes-insectes, saponines, sapogénines, allomones. yam (Dioscoreaceae) leaves.

The Guadeloupean leaf-cutting ant, Acromyrmex octospinosus, is a severe defoliator of yam plantations but some species of Dioscorea are disregarded. This behaviour was verified under field and laboratory conditions and confirmed the unpalatability of the Dioscorea cayenensis species group. The resistance of these leaves was chemical in origin, and the responsible compounds were not repellent but antifeedant. They were methanolextractible and the saponin group seemed to be involved in the plant's self-protection.

Additional key words : Fungus growing ant, Dioscorea, leaf-cutting ant, plants-insects relations, saponins, sapogenins, allomones.

\section{INTRODUCTION}

Les fourmis champignonnistes (Attini) des genres Atta et Acromyrmex sont parmi les plus importants ravageurs des plantes cultivées ou spontanées du monde tropical américain (CRAMER, 1967). Ces fourmis cultivent un basidiomycète symbiotique sur un substrat végétal frais constitué de fleurs et de feuilles découpées dans l'environnement du nid. WEBER (1966) estime que les fourmis ne consomment que leur symbiote et ces insectes sont généralement considérés comme " monophages ". L'ingestion de sève des plantes découpées est cependant démontrée par LITTLEDYKE \& CHERRETT (1976). Au vu de l'activité de découpe des végétaux, ces fourmis peuvent être consi- dérées comme "polyphages " au sens écologique du terme (CHERRETT, 1972). En effet une très grande variété de plantes est exploitée (WEBER, 1966 ; CHERRETT, 1968). Face à cette large gamme de matériel végétal, les attines présentent cependant des préférences (AMANTE, 1967 ; CHERRETT, 1968 ; ROCKWOOD, 1976, 1977 ; POLlard et al., 1983) ; certaines espèces paraissent résistantes aux attaques alors que des taxons proches s'avèrent particulièrement sensibles.

En Guadeloupe, les dégâts occasionnés par l'attine, Acromyrmex octospinosus (Reich), sont surtout dommageables à la polyagriculture traditionnelle. Les ignames (Dioscoréacées), importantes plantes locales à tubercules, sont particulièrement attaquées. Toutefois, parmi les 4 espèces cultivées (Dioscorea bulbifera L. ; 
$D$. alata L. ; D. trifida L. et $D$. cayenensis sousespèces cayenensis Lam. et rotundata Poir.), l'igname jaune (D.c. cayenensis) présente une nette résistance à la défoliation.

Cette étude tente de déterminer les causes de l'inappétence d'Acromyrmex octospinosus pour l'igname jaune (D.c. cayenensis).

\section{MATÉRIEL ET MÉTHODES}

\section{A. Matériel animal}

Les expériences sont conduites sur des nids récoltés dans l'île de la Grande-Terre (Est-Guadeloupe) et gardés au laboratoire à $25-30{ }^{\circ} \mathrm{C}, 70-80$ p. $100 \mathrm{HR}$. Ils contiennent tous une reine féconde et la meule fongique a un volume d'environ 0,5 à $1 \mathrm{l}$. En dehors des périodes d'expériences, ces nids sont approvisionnés quotidiennement avec du matériel végétal frais, varié et apprécié.

\section{B. Matériel végétal}

L'étude porte sur 5 espèces de Dioscoréacées, cultivées en plein champ et n'ayant subi aucun traitement phytosanitaire: $D$. cayenensis cayenensis (igname jaune), D. cayenensis rotundata (grosse caille), $D$. alata (igname blanche), $D$. trifida (cousse-couche) et D. bulbifera (adon).

\section{Protocoles expérimentaux}

1) La préférence des fourmis pour les différentes espèces d'igname est vérifiée au laboratoire : 2 lots de feuilles entières sont proposés à 3 nids. Un lot est toujours formé de feuilles d'adon, espèce de référence et le $2^{\mathrm{e}}$ lot comprend des feuilles d'une des 4 autres espèces d'igname, chacune étant expérimentée pendant 3 à 4 périodes de $4 \mathrm{~h}$ sur chacun des nids. A la fin d'une période, la masse de feuilles découpées est mesurée par différence de pesées pour chaque lot. La mesure est corrigée des pertes dues à l'évapotranspiration (grâce à un lot témoin de feuilles laissées dans la pièce d'expérience) et rapportée à la référence " adon ».

A la fin de cette $1^{\text {re }}$ série d'expériences, les 3 nids sont approvisionnés, ad libitum, uniquement avec des feuilles d'igname jaune.

2) Le test comportemental consiste à suivre la prise de confetti par les ouvrières (confetti de végétal ou de papier filtre imbibés d'extraits) déposés sur une grille de test dessinée sur le plateau d'affouragement des nids. Cette grille (carré latin $5 \times 5$ ) permet de déposer simultanément jusqu'à 5 séries différentes de confetti. Chaque prise est immédiatement remplacée par un confetti de même nature. Pour chaque série, les confetti prélevés sont comptabilisés soit sur une période de $20 \mathrm{mn}$, soit dès que 50 confetti sont prélevés dans une série ; les résultats étant alors interpolés jusqu'à $20 \mathrm{mn}$. Un essai complet comprend de 2 à 4 répétitions sur 5 nids différents. Afin de diminuer l'influence des variations de l'intensité d'affouragement (variable selon les nids ou les jours d'expériences), les pourcentages de prises pour chaque série sont considérés par rapport au nombre total de prises.
Après la transformation angulaire, arc sinus racine (normalisation de la distribution des données), les résultats subissent une analyse de variance à 2 facteurs (modèle mixte croisé), suivie d'un test de NEWMAN \& KEULS.

\section{Extractions chimiques}

Les extractions chimiques sont effectuées sur 2 espèces d'igname : $D$. bulbifera et D.c. cayenensis. Elles sont réalisées par extractions successives au soxhlet avec des solvants de polarité croissante (hexane, dichlorométhane et méthanol) et terminées par une infusion dans l'eau bouillante. Chaque extrait, évaporé à sec (sous pression réduite), est repris dans une quantité connue de solvant afin d'être déposé sur des confetti en papier filtre. Une quantité d'extrait provenant d'une masse équivalente de feuille peut ainsi être définie.

Dans un $1^{\text {er }}$ temps et pour chaque solvant, les extraits des 2 espèces d'igname sont comparés avec le protocole expérimental $\mathrm{n}^{\circ} 2$ précédent. Une série de confetti témoins traités avec le solvant seul est également déposée sur la grille.

Dans un $2^{\mathrm{e}}$ temps et pour chaque solvant, l'extrait de D.c. cayenensis est comparé à lui-même, additionné d'une substance appétante (solution sucrée au goût d'orange, TANG(1), à $50 \mathrm{~g} / 1$ ). Dans ce cas, 2 séries de confetti témoins sont réalisées simultanément : l'une est traitée avec le solvant seul et l'autre avec le solvant additionné de la solution appétante.

\section{E. Analyse chimique et dosage des sapogénines}

Les extraits méthanoliques des 2 espèces, $D$. bulbifera et $D$.c. cayenensis sont analysés en chromatographie couche mince (plaque de gel de silice, MERCK réf. 10401), produit éluant, mélange dichlorométhaneméthanol (85-15). Différents groupements chimiques sont caractérisés : révélations à l'iode et avec les réactifs de DRAGENDORF (alcaloïdes et autres composés azotés), à la vaniline (phénols, stéroïdes) et au trichlorure d'antimoine 300 p. 100 dans de l'acide chlorhydrique concentré (saponines et sapogénines, BRAIN \& HARDMAN, 1968).

Une comparaison des saponines et sapogénines totales est réalisée sur les 5 espèces d'igname étudiées. L'hydrolyse des saponines et l'extraction des sapogénines sont effectuées selon la méthode de MORRIS et al. (1958). Les extraits sont chromatographiés (gel de silice) avec du chlorure de méthylène $(20 \mathrm{p}$. 100) dans du benzène comme éluant. Les chromatogrammes sont révélés selon la méthode de BRAIN \& HARDMAN (1968). Le dosage des sapogénines dans les 5 extraits est réalisé selon la méthode de SOFOWORA \& HARDMAN (1974), la diosgénine étant utilisée comme standard.

\section{RÉSULTATS}

Les quantités relatives de feuilles des 5 espèces d'igname, découpées par les fourmis (selon le protocole $\mathrm{n}^{\circ}$ 1) sont présentées à la figure 1. Au laboratoire, les nids présentent des préférences identiques à celles observées sur le terrain : une récolte importante 

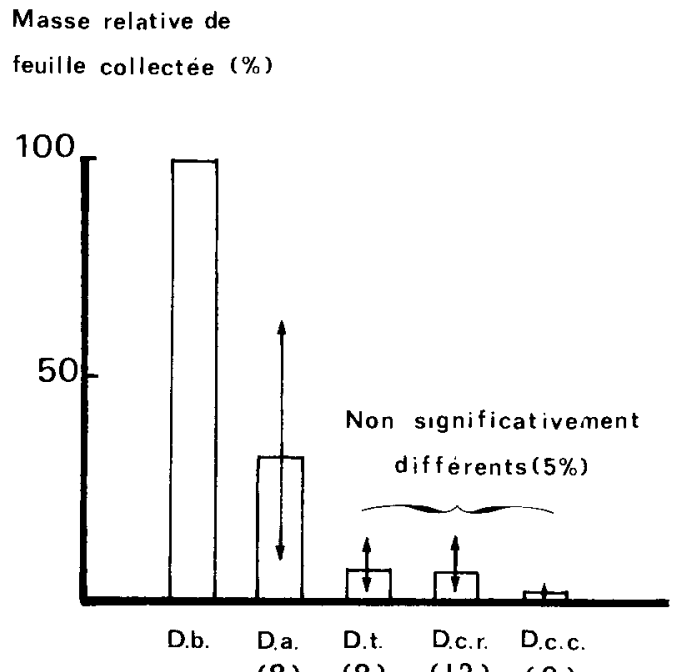

(8) (8) (12) (9)

Figure 1

Masse relative de feuille collectée par $\mathrm{A}$. octospinosus pour les 5 espèces d'igname. Les données sont exprimées en pourcentage de D. bulbifera. Les barres verticales représentent l'intervalle de confiance à $5 \%$; les nombres de répétitions sont entre parenthèses (analyses statistiques effectuées sur les transformées angulaires).

Relative weight of the leaf fragments collected by A. octospinosus from 5 yam species. Relative weight expressed in percent of D. bulbifera. Vertical bars represent the confidence interval $(5 \%)$. Numbers of replicates in parentheses. Statistical analysis on angular transformed data.

$D . b .=$ Dioscorea bulbifera $; D . a .=\mathrm{D}$. alata $; D . t .=\mathrm{D}$. trifida $;$ $D$. $c . r .=\mathrm{D}$. cayenensis rotundata ; D. c. $c .=\mathrm{D}$. cayenensis cayenensis.

de $D$. bulbifera et une découpe quasi nulle des feuilles de D.c. cayenensis.

L'approvisionnement en continu avec les seules feuilles de D.c. cayenensis aboutit irrémédiablement à la mort des nids. Deux meules fongiques, d'un volume initial approximatif de $500 \mathrm{ml}$, ont été éliminées en 9 et $12 \mathrm{j}$. Un $3^{\mathrm{e}}$ nid, plus important (volume compris entre 1 et $1,5 \mathrm{l}$ ) a dépéri en $27 \mathrm{j}$. La disparition du jardin à champignon paraît être directement liée à l'annulation de l'affouragement. L'observation du comportement des ouvrières au cours de cette expérience révèle que les feuilles de D.c. cayenensis ne contiennent pas de répulsifs ( $=$ substances qui agissent à distance et empêchent toute approche de l'insecte). En effet, les grandes ouvrières, chargées de la découpe du matériel végétal, peuvent être observées en nombre important sur les feuilles d'igname jaune. Certaines entreprennent la découpe du végétal, mais, la plupart du temps, sans mener cette opération à terme. Les rares morceaux prélevés sont abandonnés à l'intérieur du nid, très peu étant intégrés dans la meule fongique. Ce comportement semble être caractéristique d'une réponse à la présence d'antiappétants au sens de VIGNERON (1978): action par contact, inhibition de l'alimentation mais sans mort ni répulsion de l'insecte.

La qualité physique des feuilles pourrait cependant rendre difficile la mécanique de la découpe par les ouvrières. Cette hypothèse est soumise à un test comportemental utilisant des confetti de végétaux. L'étape initiale, c'est-à-dire la découpe de la feuille par les ouvrières, compte tenu des caractéristiques physiques, est alors évitée. Les différentes espèces d'igname sont expérimentées de front sur 5 nids (4 répétitions). Les résultats (en pourcentages de prises anamorphosés pour chaque série) montrent que le facteur nid n'est pas significatif et permet de retenir les moyennes pour chaque espèce d'igname (tabl. 1). Au seuil de $5 \mathrm{p}$. 100 , le test de Newman \& Keuls définit 4 groupes de préférence décroissante: $D$. bulbifera, $D$. alata, $D$. trifida et une dernière classe regroupant les 2 sousespèces de $D$. cayenensis : cayenensis et rotundata. Ces résultats confirment que le choix des fourmis n'est pas lié à une différence de qualité physique entre les feuilles d'ignames.

La présence de composés antiappétants est recherchée dans les feuilles de $D$. cayenensis et parallèlement dans celles de D. bulbifera et D.c. cayenensis dont chaque extrait est soumis au test éthologique. L'analyse des données transformées indique que le facteur nid n'est pas significatif. La comparaison des prises est observée (tabl. 2) entre les extraits des

\section{TABLEAU 1}

Préférence d'A. octospinosus pour les 5 espèces d'igname. Les données (pourcentages de prises, par rapport à tous les confetti végétaux prelevés) pour chaque espece représentent les moyennes de 20 répétitions. Les moyennes indicées de la même lettre ne sont pas significativement différentes $(P<0,05$ test de NEWMAN \& KEULS réalisé sur les données après transformations angulaires).

Preference of A. octospinosus for 5 species of yam. Data for each species as \% of all confetti picked up and means of 20 replicates. Means with the same letter not significantly different (S.N.K. on angular transformed data, $P<0.05$ ).

\begin{tabular}{lccccc}
\hline \hline $\begin{array}{c}\text { Espèces } \\
\text { d'igname }\end{array}$ & bulbifera & alata & trifida & $\begin{array}{c}\text { cay. } \\
\text { rotundata cayenensis }\end{array}$ \\
\hline $\begin{array}{l}\text { Moyenne des } \\
\text { pourcentages } \\
\text { de prises }\end{array}$ & $58,3^{\mathrm{a}}$ & $26,1^{\mathrm{b}}$ & $7,6^{\mathrm{c}}$ & $3,6^{\mathrm{d}}$ & $2,3^{\mathrm{d}}$ \\
\hline $\begin{array}{l}\text { Intervalle de } \\
\text { confiance }(5 \%)\end{array}$ & 52,6 & 22,6 & 5,1 & 2,1 & 1,5 \\
\hline \hline
\end{tabular}

TABLEAU 2

Préférence d'A. octospinosus pour les extraits de 2 espèces d'igname obtenus avec différents solvants. Pour chaque solvant, les données sont les pourcentages de prises, par rapport à tous les confetti prélevés, pour chaque type d'extrait et représentent une moyenne de 10 à 20 répétitions. Pour chaque ligne, les moyennes indicées d'une même lettre ne sont pas significativement différentes $(P<0,05$ test de NEWMAN \& KEULS, réalisé sur les données après transformation angulaire).

Preference of A. octospinosus for extracts of 2 yam species obtained with 4 different solvents. For each solvent, data as \% of all confetti picked up, each the mean of 10 to 20 replicates. For each solvent, means with the same letter not significantly different (S.N.K. on angular transformed data, $P<0.05$ ).

\begin{tabular}{lccc}
\hline \hline & $\begin{array}{c}\text { Témoins } \\
\text { solvants }\end{array}$ & $\begin{array}{c}\text { Extraits de } \\
\text { D. cayenensis }\end{array}$ & $\begin{array}{c}\text { Extraits de } \\
\text { D. bulbifera }\end{array}$ \\
\hline Hexane & $6,8^{\mathrm{a}}$ & $1,0^{\mathrm{b}}$ & $91,9^{\mathrm{c}}$ \\
\hline Dichlorométhane & $4,5^{\mathrm{a}}$ & $6,7^{\mathrm{a}}$ & $87,2^{\mathrm{b}}$ \\
\hline Méthanol & $19,1^{\mathrm{a}}$ & $2,4^{\mathrm{a}}$ & $75,1^{\mathrm{b}}$ \\
\hline Eau & $33,9^{\mathrm{a}}$ & $1,8^{\mathrm{b}}$ & $2,5^{\mathrm{b}}$ \\
\hline \hline
\end{tabular}


2 espèces d'igname et le témoin. Pour les 3 solvants (hexane, dichlorométhane et méthanol), la prise des confetti imprégnés d'extraits de $D$. bulbifera est très significativement supérieure à celle obtenue avec les extraits de D.c. cayenensis, elle-même non significativement différente (dichlorométhane et méthanol) ou inférieure (hexane) à la prise des confetti témoins. Pour l'extrait aqueux des 2 espèces d'igname, la prise est significativement inférieure à celle des confetti témoins. Cette expérience indique soit la présence de substances antiappétantes dans les extraits organiques de D.c. cayenensis, soit celle d'attractifs dans les extraits de $D$. bulbifera.

Les tests comportementaux correspondants sont effectués sur les extraits organiques de D.c. cayenensis ainsi que sur ces mêmes extraits complétés avec une substance appétante. Les résultats sont reportés au tableau 3. L'extrait méthanolique additionné de la solution appétante n'entraîne pas d'augmentation significative de la prise des confetti. Cet extrait contient des substances antiappétantes. Avec les 2 autres solvants (hexane et dichlorométhane), si les extraits contiennent aussi des substances antiappétantes, celles-ci sont moindres soit en quantité, soit en efficacité. L'addition de la substance appétante à l'extrait hexanique induit une augmentation significative de la prise des confetti. Cette dernière reste cependant plus faible que la prise des confetti témoins appétants. Pour l'extrait au dichlorométhane, l'addition de la solution appétante n'augmente pas significativement la prise des confetti. Celle-ci n'est pas non plus statistiquement différente de la prise des confetti témoins appétants. Dans le cas du dichlorométhane, le solvant contient peut-être des impuretés qui interfèrent.

L'analyse en chromatographie couche mince réalisée sur les extraits méthanoliques des 2 espèces d'igname ne révèle aucune différence notable pour les groupements fonctionnels réagissant à l'iode, les alcaloïdes et les phénols. Par contre, pour les saponinessapogénines, 2 taches importantes sont visualisées dans l'extrait de D.c. cayenensis et restent absentes dans celui de $D$. bulbifera.

TABLEAU 3

Préférence d'A. octospinosus pour différents extraits, seuls ou additionnés d'un appétant. Pourcentages de prises pour chaque solvant par rapport à tous les confetti prélevés (moyennes de 10 à 15 répétitions). Pour chaque ligne, les moyennes indicées d'une même lettre ne sont pas significativement différentes $(P<0,05$ test de NEWMAN \& KEULS réalisé sur les données après transformation angulaire).

Preference of A. octospinosus for different extracts, pure or mixed with a feeding stimulant. Data as \% of all confetti picked up, each the mean of 10 to 15 replicates. For each solvent, means with the same letter not significantly different (S.N.K. on angular transformed data, $P<0.05$ ).

\begin{tabular}{lcccc}
\hline & $\begin{array}{c}\text { Extraits de } \\
\text { D. c. caye- } \\
\text { nensis }\end{array}$ & $\begin{array}{c}\text { Témoins } \\
\text { solvants }\end{array}$ & $\begin{array}{c}\text { Extraits de } \\
\text { D. caye- } \\
\text { nensis } \\
\text { appétant }\end{array}$ & $\begin{array}{c}\text { Témoins } \\
\text { solvants } \\
\text { appétant }\end{array}$ \\
\hline Hexane & $0,6^{\mathrm{a}}$ & $1,3^{\mathrm{a}}$ & $37,7^{\mathrm{b}}$ & $59,2^{\mathrm{c}}$ \\
\hline Dichlorométhane & $10,6^{\mathrm{ab}}$ & $1,1^{\mathrm{a}}$ & $27,5^{\mathrm{bc}}$ & $53,1^{\mathrm{c}}$ \\
\hline Méthanol & $5,1^{\mathrm{ab}}$ & $1,4^{\mathrm{a}}$ & $10,6^{\mathrm{b}}$ & $79,5^{\mathrm{c}}$ \\
\hline \hline
\end{tabular}

L'hydrolyse acide des saponines en sapogénines et l'extraction de celles-ci à l'éther de pétrole sont réalisées sur les feuilles des 5 espèces d'igname. Le dosage des sapogénines selon la méthode de SOFOWORA \& HARDMAN (1974) ne permet pas de les mettre en évidence dans les extraits de $D$. bulbifera, $D$. alata et D. trifida. Par contre, les 2 autres espèces possèdent une concentration en sapogénines (en équivalent diosgénine) égale à $0,73 \mathrm{mg} / \mathrm{g}$ de poids sec de feuilles chez D.c. rotundata et à $0,25 \mathrm{mg} / \mathrm{g}$ de $\mathrm{PS}$ pour $D$.c. cayenensis. La chromatographie couche mince de ces extraits confirme ces résultats : une tache commune de sapogénines est visualisée pour les 5 espèces d'igname, seules D.c. cayenensis et D.c. rotundata présentent 2 taches supplémentaires (fig. 2).

\section{DISCUSSION}

Les interactions complexes entre les insectes phytophages et les plantes sont les résultats d'un processus long et continu. Toutes les tendances de cette évolution parallèle peuvent être divisées en 2 aspects principaux (BECK, 1965 ; LEVIN, 1976) : développement par les plantes de mécanismes de défense ; adaptation des insectes phytophages contrecarrant ces derniers. Le $1^{\text {er }}$ aspect peut être assuré par les plantes de différentes manières :

- texture et structure anatomique (poils, épines...) de protection ;

- $\mathrm{pH}$, pression osmotique de la sève impropre pour l'insecte ;

- absence de nutriments exigés par le ravageur ;

- présence de produits métaboliques secondaires qui peuvent affecter le développement de l'insecte

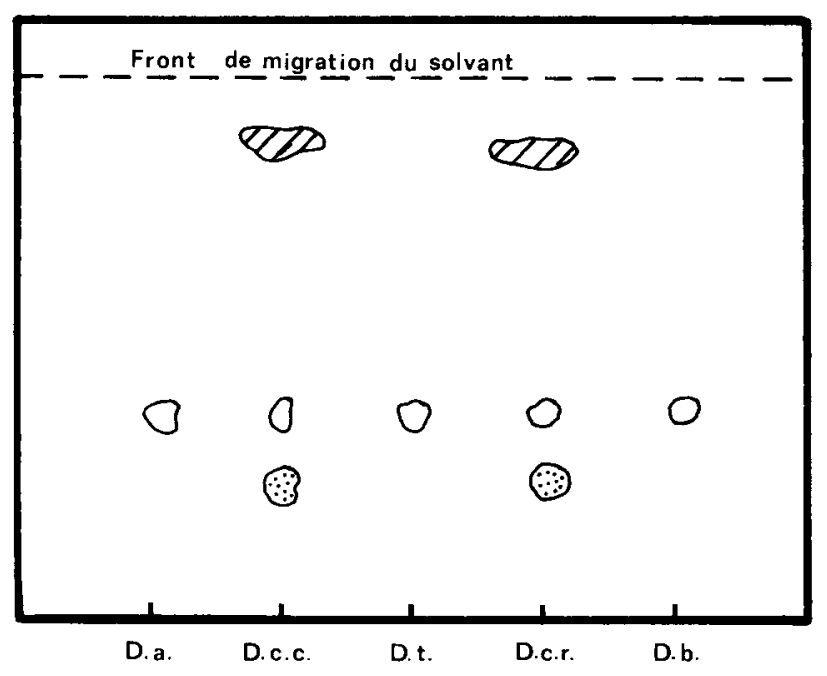

Figure 2

Chromatographie couche mince après hydrolyse des saponines et extraction des sapogénines des feuilles de 5 espèces d'igname par la méthode de MORRIS et al. (1958). Les sapogénines sont révélées par la méthode de BRAIN \& HARDMAN (1968).

Thin layer chromatography following hydrolysis of saponins and extraction of sapogenins from 5 yam species leaves according to the method of MORRIS et al. (1958). Sapogenins revealed by the method of BRAIN \& HARDMAN (1968).

D. $a .=$ D. alata ; D. c. c. $=$ D. c. cayenensis ; D. $t .=$ D. trifida ; D. c. $r .=$ D. c. rotundata $; D . b .=$ D. bulbifera. 
(insecticides naturels), complexer les nutriments (protéines en particulier)...

Ces substances secondaires intervenant dans les relations interspécifiques (substances allélochimiques) appartiennent à la catégorie des allomones (avantage adaptatif à l'organisme qui les produit ; rôle protecteur ou défensif). Néanmoins sur la base de cette classification fonctionnelle, une même substance produite par une plante pourra être rangée dans les allomones, kairomones ou phéromones selon les espèces et les relations envisagées (PESSON, 1980).

Une terminologie spécifique est adoptée pour les substances allélochimiques intervenant dans le comportement d'alimentation des insectes (DETHIER et al., 1960 ; MUNAKATA, 1975). Deux étapes principales sont considérées : reconnaissance et localisation de la plante hôte ; début et poursuite de l'alimentation.

Pour la $1^{\text {re }}$ étape, on distingue les attractifs (l'insecte répond au stimulus en s'orientant vers la source alimentaire) et les répulsifs (l'insecte s'éloigne de la nourriture). Ces substances agissent à distance et sont volatiles. En ce qui concerne la $2^{\mathrm{e}}$, on définit les appétants (stimulant la prise de nourriture) et les antiappétants (inhibant l'alimentation), substances qui agissent par contact.

Dans le cas des fourmis attines, les relations avec les plantes sont compliquées par la culture du champignon symbiotique sur le substrat végétal. Ce symbiote est la principale source de nourriture de l'insecte (WEBER, 1972), cependant il est démontré que les ouvrières ingèrent également la sève des plantes découpées (LITTLEDYKE \& CHERRETT, 1976). Aussi le refus de certains végétaux peut avoir des bases fonctionnelles différentes. Il est possible que les fourmis délaissent toute plante présentant des substances toxiques pour le champignon ou sur lesquelles la croissance de ce dernier serait freinée (fongicides, fongistatiques). Les fourmis goûtent, au niveau des palpes buccaux, la sève des végétaux découpés et certaines plantes insecticides sont ainsi évitées. Cependant CHERRETT (1980) considère que l'alliance fourmichampignon rend possible une plus large polyphagie ; chaque partenaire pouvant détruire les systèmes de défense des plantes plus spécifiquement dirigés contre l'autre.

Toutefois certains végétaux sont résistants aux attines et les différents mécanismes classiques de défense des plantes paraissent efficients. Ainsi les qualités physiques des feuilles peuvent être à l'origine d'une difficulté mécanique de découpe par la fourmi. WALLER (1982) montre que les feuilles de Berberis trifoliata Hartw. (Berberidaceae) ne sont pas répulsives mais trop dures pour être découpées par Atta texana (Buckley). STRADLING (1978) détermine que le latex constitue une barrière physico-chimique à la découpe des plantes laticifères. La teneur en eau ou en nutriments facilement accessibles peut aussi être un facteur du choix de la fourmi : ROCKWOOD (1976) suggère que le comportement sélectif d'affouragement des ouvrières d'Atta dans la nature tend à minimiser l'influence des substances chimiques secondaires des plantes sur le champignon pour favoriser dans le même temps le contenu nutritionnel. BOWERS \& PORTER (1981) estiment que les attines choisissent les feuilles les plus aqueuses. Enfin certains composés chimiques végétaux peuvent être autant de facteurs dissuasifs. LITTLEDYKE \& CHERRETT (1978) démontrent, au laboratoire, que les extraits de vieilles feuilles de plusieurs espèces végétales inhibent la découpe par Atta cephalotes (L) et Acromyrmex octospinosus, les substances inhibitrices étant concentrées dans la fraction lipidique des extraits. HUBBELL \& WIEMER (1983) montrent que les feuilles de l'arbre tropical Hymenaea courbaril L. ne sont pas découpées par les Atta du fait de la présence d'un répulsif volatil, l'époxide de caryophyllène, qui ne serait pas toxique pour l'insecte mais plutôt fongicide. CHEN \& WIEMER (1984) rapportent également qu'un répulsif volatil, le trans- $\beta$ ocimène, est à la base de la protection d'Astronium graveolens (Anacardiaceae) contre $A$. cephalotes.

\section{CONCLUSION}

Les résultats mentionnés dans cette étude apportent quelques nouveaux arguments dans ce sens. A l'intérieur d'un même genre végétal, $A$. octospinosus ravage certaines espèces alors que d'autres sont totalement délaissées. A notre connaissance, c'est la $1^{\text {re }}$ fois qu'il est montré que les nids d'une attine peuvent péricliter jusqu'à disparaître par refus d'affourager sur le végétal mais régulièrement à sa disposition. La résistance de D.c. cayenensis à $A$. octospinosus est d'origine chimique. Cependant les composés en cause ne sont pas répulsifs et doivent être classés parmi les antiappétants. Les saponines-sapogénines paraissent être à l'origine de cette résistance et leur structure chimique sera précisée dans un travail ultérieur. Les saponines sont des glucosides dont la partie aglycone (= sapogénine) est de nature terpénoïde (penta- ou tétracyclique), stéroïde ou stéroïde-alcaloïde (TSCHESCHE \& WULFF, 1973). Ces composés, synthétisés presque exclusivement par les végétaux supérieurs, sont caractérisés par des propriétés biologiques dont la principale est leur toxicité pour les champignons (DEFAGO, 1977). De nombreux auteurs ont souligné le rôle des saponines dans le mécanisme de défense des plantes lors d'agressions par des phytopathogènes (AlLEN \& KUC, 1968 ; MAIZEL et al., 1964 ; SCHLOSSER, 1971, 1975 ; RODDICK, 1974 ; DEFAGO et al., 1975). Toutefois, certains résultats n'ont pas confirmé cette hypothèse (FRANK et al., 1975). Ces composés seraient aussi à l'origine de la résistance de certaines plantes contre les insectes (DAHLMAN \& HIBBS, 1967 ; STRUCKOW \& LOW, 1961).

La propriété fongicide attribuée aux saponines permet de poser, pour le cas particulier des fourmis attines, une question fondamentale : la co-évolution du complexe ectosymbiotique fourmi-champignon avec les plantes supérieures a-t-elle abouti à la perception et la reconnaissance par l'insecte des composés toxiques pour son partenaire basidiomycète? Les recherches, poursuivies dans ce sens, devraient permettre d'apporter de nouveaux éléments pour la compréhension des relations particulières entre les attines et les plantes. Enfin, l'étude des mécanismes de protection des ignames est d'un intérêt agronomique évident : leur connaissance permet d'imaginer de nouvelles méthodes de protection des cultures trop souvent ravagées par les 
attines : amélioration de variétés d'ignames par génie génétique (inclusion des gènes de synthèse des antiappétants), protection d'autres cultures sensibles par pulvérisation sur les feuillages de composés antiappétants.

Reçu le 30 juillet 1984. Accepté le 3 janvier 1985.

\section{REMERCIEMENTS}

Une partie de ces travaux a été effectuée avec l'aide des stagiaires suivants: $\mathbf{L}$. Durour, M. Guerrier et V. Corradin.

Ces travaux ont été financés par la CORDET (contrat Agronomie $\left.n^{\circ} \mathrm{C} 101,1983\right)$ et seront achevés grâce à la même aide $\mathrm{n}^{\circ} \mathrm{C} 402$ (1984). Nous remercions le Dr. Degras L. (I.N.R.A., Station d'Amélioration des Plantes, Guadeloupe) pour le matériel végétal mis à notre disposition et pour nous avoir fait partager ses connaissances sur les Dioscoreacées.

\section{RÉFÉRENCES BIBLIOGRAPHIQUES}

Allen E. H., Kuc J., 1968. $\alpha$-solanine and $\alpha$-chaconine as fungitoxic compounds in extracts of Irish potato tubers. Phytopathology, 58, 776-781.

Amante E., 1967. A formiga sauva Atta capiquara praga das pastagens. Biologico, 33, 113-120.

Beck S. D., 1965. Resistance of plants to insects. Annu. Rev. Entomol., 10, 207-232.

Bowers M. A., Porter S. D., 1981. Effect of foraging distance on water content of substrate harvested by Atta columbica (Guerin). Ecology, 62, 273-275.

Brain K. R., Hardman R., 1968. An improved method of densitometric thin layer chromatography as applied to the determination of sapogenins in Dioscorea tubers. J. Chromatog., 38, 355-363.

Chen T. K., Wiemer D. F., 1984. A volatile leafcutter and repellent from Astronium graveolens. Naturwissenschaften, 71, 97-98.

Cherrett J. M., 1968. The foraging behaviour of Atta cephalotes L. (Hymenoptera, Formicidae). I : Foraging pattern and plant species attacked in tropical rain forest. J. anim. Ecol., 37, 387-403.

Cherrett J. M., 1972. Some factors involved in the selection of vegetable substrate by Atta cephalotes (L.) (Hymenoptera, Formicidae) in tropical rain forest. J. anim. Ecol., 41, 647-660.

Cherrett J. M., 1980. Possible reasons for the mutualism between leaf-cutting ants (Hymenoptera, Formicidae) and their fungus. Biol. Ecol. Méditerr., 7, 113-122.

Cramer H. H., 1967. Plant protection and world crop production. Pflanzenschutz Nachrichtenbl. Bayer, 20, 1-524.

DahIman D. L. Hibbs E. T., 1967. Response of Empoasca fabae to tomatine, solanine, leptine 1 , tomatidine, soladine and demissidine. Ann. entomol. Soc. Am., 60, 732-740.

Defago G., 1977. Rôle des saponines dans la résistance des plantes aux maladies fongiques. Ber. Schweitz. Bot. Ges., 87, 79-132.

Defago G., Memmen K. F., Kern H., 1975. Influence du cholestérol et des saponines sur le pouvoir pathogène du Pythium paroecandrum. Phytopathol. Z., 83, 167-184.

Dethier V. G., Barton-Browne L., Smith C. N., 1960. The designation of chemicals in terms of the responses they elicit from insects. J. Econ. Entomol., 53, 134-136.

Frank J. A., Wilson J. M., Webb R. E., 1975. The relationship between glycoalkaloids and disease resistance in potatoes. Phytopatho$\log y, 65,1045-1049$.

Hubbell S. P., Wiemer D. F., 1983. Host plant selection by an attine ant, p. 133-154. In P. Jaisson : "Social insects in the tropics ». Univ. Paris Nord, II, $252 \mathrm{p}$.

Levin D. A., 1976. The chemical defenses of plants to pathogens and herbivores. Annu. Rev. Ecol. Syst., 7, 121-159.

Littledyke M., Cherrett J. M., 1976. Direct ingestion of plant sap from cut leaves by the leaf-cutting ants Atta cephalotes (L.) and Acromyrmex octospinosus (Reich) (Formicidae, Attini). Bull. Ent. Res., 66, 205-217.
Littledyke M., Cherrett J. M., 1978. Defence mechanisms in young and old leaves against cutting by the leaf-cutting ants Atta cephalotes (L.) and Acromyrmex octospinosus (Reich) (Hymenoptera, Formicidae). Bull. Ent. Res., 68, 263-271.

Maizel J. V., burkhardt H. J., Mitchell H. K., 1964. Avenacin, an antimicrobial substance isolated from Avena sativa. I. Isolation and antimicrobial activity. Biochemistry, 3, 424-426.

Morris M. P., Roark B. A., Cancel B., 1958. Simple procedure for the routine assay of Dioscorea tubers. Agric. Food Chem., 6, 856 858.

Munakata K., 1975. Insect antifeeding substances in plant leaves. Pure Appl. Chem., 42, 57-66.

Pesson P., 1980. A propos de l'instinct botanique des insectes : un aspect de la co-évolution des plantes et des insectes. Ann. Soc. Entomol. Fr., 16, 435-452.

Pollard G. V., Riley R., Wattie E., 1983. Preliminary investigations on the selection of citrus species by the leaf-cutting ant, Acromyrmex octospinosus (Reich) (Formicidae, Attini). Trop. Agric. (Trinidad), 60, 282-285.

Rockwood L. L., 1976. Plant selection and foraging patterns in two species of leaf-cutting ants (Atta). Ecology, 57, 48-61.

Rockwood L. L., 1977. Foraging patterns and plant selection in Costa Rican leaf-cutting ants. J. N. Y. Entomol. Soc., 85, 222-233.

Roddick J. G., 1974. The steroidal glycoalcaloid $\alpha$-tomatine. Phytochemistry, 13, 9-25.

Schlosser E., 1971. Cyclamin, an antifungal resistance factor in cyclamen species. Acta Phytopathol. Acad. Sci. Hung., 6, 85-95.

Schlosser E., 1975. Role of saponins in antifungal resistance. III. Tomatine dependent development of fruit rot organisms on tomato fruits. Z. Pflkrankh. Pflschutz., 82, 476-484.

Sofowora E. A., Hardman R., 1974. Chromatographic detection and spectrophotometric determination of diosgenin and other $\Delta 5$ sapogenins in crude plant extracts. Planta Med., 26, 385-390.

Stradling D. J., 1978. The influence of size on foraging in the ant, Atta cephalotes, and the effect of some plant defence mechanisms. J. Anim. Ecol., 47, 173-188.

Struckow B., Low I., 1961. The effect of some solanum alkaloid glycosides on the potato beetle. Entomol. Exp. appl., 4, 133-142.

Tschesche R., Wulff G., 1973. Chemie und Biologie der Saponine. Fortschr. Chem. Org. Naturst., 30, 462-606.

Vigneron J. P., 1978. Substances antiappétantes d'origine naturelle. Ann. Zool. Ecol. Anim., 10, 663-694.

Waller D. A., 1982. Leaf-cutting ants and avoided plants : defences against Atta texana attack. OEcologia, 52, 400-403.

Weber N. A., 1966. Fungus-growing ants. Science N. Y., 153, $587-$ 604.

Weber N. A., 1972. Gardening ants : the Attines. Mem. Am. phil. Soc., 92, 1-146. 